\title{
Staphylococcus felis
}

National Cancer Institute

\section{Source}

National Cancer Institute. Staphylococcus felis. NCI Thesaurus. Code C86759.

A species of facultatively anaerobic, Gram positive, cocci shaped bacteria in the phylum Firmicutes. This species is positive for alkaline phosphatase, urease and catalase and negative for coagulase and oxidase. This species can ferment mannose, and trehalose but not maltose or ribose. S. felis is pathogenic in cats causing external otitis, cystitis, abscesses and other infections. 